\title{
Antagonistic effects of drought and sand burial enable the survival of the biocrust moss Bryum argenteum in an arid sandy desert
}

\author{
Rongliang $\mathrm{Jia}^{1}$, Yun Zhao ${ }^{1}$, Yanhong Gao ${ }^{1}$, Rong Hui ${ }^{1}$, Haotian Yang ${ }^{1}$, Zenru Wang $^{1}$, and Yixuan $\mathrm{Li}^{2}$ \\ ${ }^{1}$ Shapotou Desert Research and Experiment Station, Northwest Institute of Eco-Environment and Resources, \\ Chinese Academy of Sciences, 320 Donggang West Road, Lanzhou 730000, China \\ ${ }^{2}$ Agronomy College, Agricultural University of Shenyang, Shenyang 110866, China
}

Correspondence: Rongliang Jia (rongliangjia@163.com)

Received: 22 September 2017 - Discussion started: 1 November 2017

Revised: 24 January 2018 - Accepted: 25 January 2018 - Published: 23 February 2018

\begin{abstract}
Biocrust moss is an essential soil surface biocover. It can represent the latest succession stage among the diverse range of surface-dwelling cryptogams (e.g., cyanobacteria, green algae, and lichen, which are also referred to as biocrusts), and it can make a major contribution to soil stability and fertility in many arid sandy desert ecosystems. The soil surface represents a very large ecological niche that is poikilohydric in nature. Biocrust moss is therefore highly susceptible to drought and sand burial, which are two ubiquitous stressors in arid sandy deserts. However, little information is available regarding the mechanism by which biocrust moss can survive and flourish in these habitats when stressed simultaneously by the two stressors. The combined effects of drought and sand burial were evaluated in a field experiment using the predominant biocrust moss, Bryum argenteum Hedw., in the Tengger Desert, China. Drought was simulated by applying distilled water in three artificial rainfall regimes at 8-day intervals in spring and autumn: 4 and $6 \mathrm{~mm}$ (average rainfall, control), 2 and $3 \mathrm{~mm}$ (double drought), and 1 and $1.5 \mathrm{~mm}$ (4-fold drought), respectively. The effect of sand burial was determined by applying six treatments, i.e., sand depths of 0 (control), $0.5,1,2,4$, and $10 \mathrm{~mm}$. The four parameters of chlorophyll $a$ content, PSII photochemical efficiency, regeneration potential, and shoot upgrowth were evaluated in the moss. It was found that the combined effects of drought and sand burial did not exacerbate the single negative effects of the four parameters tested. Drought significantly ameliorated the negative effects of deep-sand burial on the retention of chlorophyll $a$ content, PSII photochemical efficiency, and the regeneration potential of B. argenteum. Sand burial diminished and even re-
\end{abstract}

versed the negative effects of drought on the maintenance of chlorophyll $a$ content, PSII photochemical efficiency, and regeneration potential. Although drought and sand burial imposed an additive negative effect on shoot upgrowth, which suggested a trade-off between growth ability and stress tolerance, their mutually antagonistic effect on the physiological vigor of $B$. argenteum provided an opportunity for the biocrust moss to overcome the two co-occurring stressors. In addition to providing a strong stress tolerance, drought and sand burial may provide an important mechanism for the biodiversity maintenance of biocrust mosses in arid sandy ecosystems.

\section{Introduction}

Drought is the most common stressor constraining biological activity in dryland ecosystems (Whitford, 2002; Huxman et al., 2004). The predicted increase in the frequency and severity of droughts is likely to generate more profound consequences for community structure and ecosystem functioning in arid and semiarid ecosystems (IPCC, 2007; Smith, 2011; Weber et al., 2016). In arid sandy ecosystems, drought generally occurs alongside another ubiquitous disturbance, sand burial, due to the lowering of the threshold friction velocities of the upper soil surface (Belnap and Gillette, 1998; Li, 2012). Sand burial can alter various physical factors such as moisture, temperature, aeration, and other aspects of the plant and soil microenvironment. It can therefore act as a filter eliminating sensitive species, and it plays a significant role in determining the composition and distribution 
of desert vegetation (Maun, 1998, 2008). Therefore, in habitats stressed simultaneously by drought and sand burial (e.g., arid desert ecosystems) throughout China and worldwide, the growth and distribution of plants is expected to be limited. This is evidenced by mobile sand dunes, with negligible vegetation cover as an extreme example.

Biocrust moss is an essential soil surface bio-cover. It can represent the latest succession stage among the diverse range of surface-dwelling cryptogams (e.g., cyanobacteria, green algae, and lichen, which are also referred to as biocrusts) and make a major contribution to soil stability and fertility in many arid and semiarid sandy desert ecosystems (Weber et al., 2016). The colonization and development of moss on the surface of a sand dune is an important biomarker denoting the ecosystem as being stable and healthy (Zhang et al., 2010). Thus, the assessment, protection, and utilization of moss is a major management priority in desert regions (Stark et al., 2004; Barker et al., 2005; Xu et al., 2009; Doherty et al., 2015).

Since the 1950s, large-scale construction and land restoration has occurred throughout the arid and semiarid sandy areas of north China, with the aims of inhibiting the harmful effects of mobile sand movement and recovering degraded ecosystems. One striking success has been the Shapotou revegetation system, which was constructed to alleviate burial stress using combined applications of wind barriers, straw checkerboards, and planting anti-drought shrubs without irrigation (Li et al., 2004, as shown in Fig. 1). Sixty years later, along with the succession of vegetation, biocrust biota have gradually colonized the area and now thrive on the previously bare soil surface, where they constitute more than $90 \%$ of the living ground cover. As a pioneer moss species, Bryum argenteum Hedw. dominates the soil surface in this system, with a coverage exceeding $70 \%$ and making a major contribution to soil stability and fertility. Its role is particularly important in areas where the sand-binding role of previously planted shrubs has weakened with time (Li et al., 2004). This phenomenon is evident throughout other sandy desert ecosystems restored by similar methods in China, where B. argenteum usually appears as the pioneer moss species. Consequently, it needs to be understood why $B$. argenteum can survive and thrive in ecosystems stressed by both drought and sand burial, enabling it to be the pioneer species.

Species that are poikilohydric in nature lack vascular support tissues but usually protrude above the soil surface to receive light for photosynthesis. Also, they are completely immobile, which prevents them from finding refuge to avoid drought stresses (Garcia-Pichel and Belnap, 1996). Bryum argenteum responds negatively to drought, despite its high desiccation tolerance (e.g., Li et al., 2014). Because it grows on the surface, $B$. argenteum is inevitably exposed to repeated sand burial of various depths. Due to its limited height above the ground surface ( 1 to $25 \mathrm{~mm}$ ) the moss can be completely buried even when the burial depth is shallow
(Jia et al., 2008). This has generated multiple organic horizons of "fossilized mosses" in areas where it has survived burial stress and barren spaces where it has not (Jia et al., 2008). Therefore, there must be a mechanism for B. argenteum to adapt to and survive this combination of stressors, although it remains poorly understood. A clear understanding of the mechanism enabling $B$. argenteum to survive the co-occurring drought and sand burial stressors in desert areas would help to explain the distribution mechanisms of this common species. It would also enable us to predict the consequences of climate change and to formulate management policies and restoration practices using biocrust moss to stabilize and rehabilitate degraded flowing sandy dunes.

Previous studies have principally focused on the individual effects of drought (Stark et al., 2004; Barker et al., 2005; Xu et al., 2009) and sand burial (Jia et al., 2008) as stressors on desert biocrust mosses, with little emphasis on their combination and even less on their interaction. Considering the different and even contrasting effects of drought and sand burial on physiology and growth, it is of interest to determine if a combination of drought and sand burial imposes a mutually antagonistic effect on the physiology and growth of B. argenteum, enabling it to survive the two co-occurring stressors. Drought is reported to protect moss from heat shock (Xu et al., 2009) and ultraviolet-B-induced damage (Turnbull et al., 2009), while sand burial has been reported to slow water loss from moss crusts (Meng et al., 2011). Therefore, our initial hypothesis is that the combination of drought and sand burial has a mutually antagonistic effect on the physiology, regeneration, and growth of $B$. argenteum. To test this hypothesis, multiple assessments of the single and combined effects of drought and sand burial stresses were made, including measurements of the chlorophyll $a$ content, PSII fluorescence, regeneration potential, and growth rate.

\section{Materials and methods}

\subsection{Study site}

The study area was located in the southeastern fringe of the Tengger Desert $\left(37^{\circ} 28^{\prime} \mathrm{N}, 105^{\circ} 00^{\prime} \mathrm{E}\right.$; elevation $\left.1339 \mathrm{~m}\right)$. It lies within the transitional zone from desert steppe to steppified desert and also represents a transitional belt between desert and oasis. Based on meteorological records from 1956 to 2003 , the mean annual temperature is $10.6^{\circ} \mathrm{C}$, with the minimum temperature being $-25.1^{\circ} \mathrm{C}$ in January and the maximum being $38.1{ }^{\circ} \mathrm{C}$ in July. The mean annual pan potential evaporation is around $3000 \mathrm{~mm}$, while the mean annual precipitation is $180.2 \mathrm{~mm}$, more than half of which falls in summer (June-August). The other three seasons typically experience more drought periods. The landscape of the study region consisted of large and dense reticulate barchan chains of sand dunes, where the predominant native plants were Hedysarum scoparium Fisch. and Agriophyllum squarrosum 


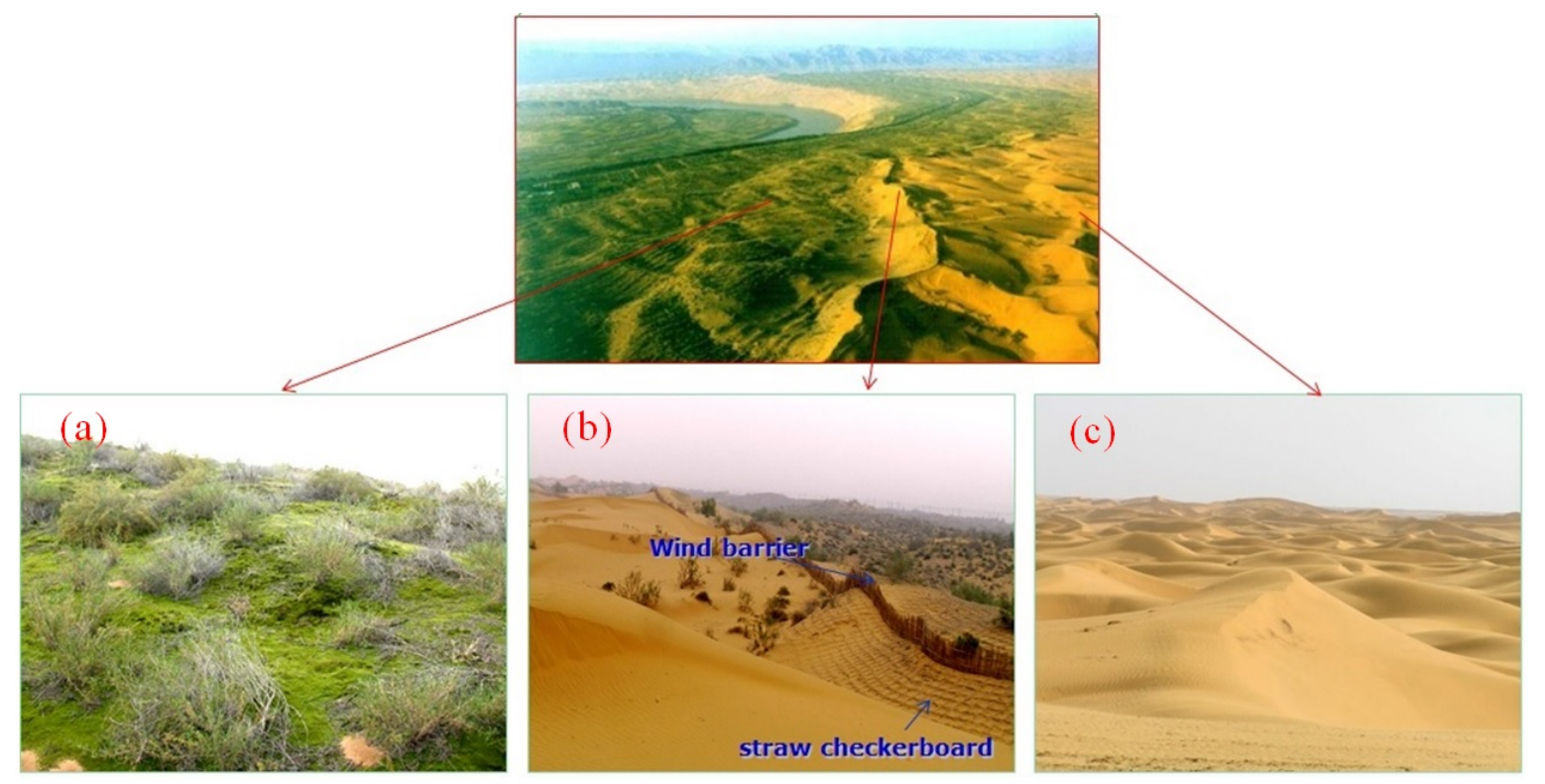

Figure 1. Main landscapes of the Shapotou region at the southeastern edge of the Tengger Desert. As a pioneer species, Bryum argenteum Hedw. has colonized and flourishes on the soil surface of the revegetation area (a); this was achieved by controlling burial stress through the combined application of wind barriers and straw checkerboards and the planting of shrubs without irrigation (b); 60 years ago, the area was characterized by shifting sand dunes (c).

Moq. that together covered about $1 \%$ of the ground surface. No biocrust was found on the surface of the mobile sand dunes (Li, 2012).

A no-irrigation vegetation system was established in 1956 to protect the Baotou-Lanzhou railway line from sand burial. It consisted of straw checkerboards as sand barriers to fix shifting dunes, with the subsequent planting of xerophytic shrub seedlings (Caragana korshinskii, Artemisia ordosica, Calligonum arborescens, etc.). The system was further expanded in 1964, 1981, and 1987. These vegetated areas were distributed parallel to the railway line, with a length of $16 \mathrm{~km}$ and a width of 1-2 km (Li et al., 2004). The initial shrub vegetation was gradually replaced by communities dominated by herbaceous plants due to the decreasing soil water content in the upper soil layers ( $\mathrm{Li}$ et al., 2004). Biocrust biota then colonized and developed on the stabilized dunes, which resulted in the surface becoming increasingly stabilized. As a pioneer species, $B$. argenteum successfully colonized the revegetated area and became widespread, with a coverage exceeding $70 \%$ on windward slopes and low-lying sand dunes. Although there is a gradual reduction in sand burial stress on the growth of $B$. argenteum as the biocrust moss becomes established, it is inevitably exposed to repeated wind-blown sand events, leading to dust burial of various depths. This burial is typically caused by two different processes that are seasonal in their severity. In spring, when the wind speed is usually the highest and drought is most severe (precipitation is the lowest), burial by wind-blown sand predominates. In summer and autumn, when the drought is slightly alleviated by higher levels of precipitation, animal activity (burrowing by ants, lizards, and rabbits) becomes important.

\subsection{Sampling and treatments}

Samples of intact moss crusts $\left(85 \mathrm{~cm}^{2}, 10 \mathrm{~cm}\right.$ thick) with $100 \%$ coverage of $B$. argenteum were randomly collected using cylindrical PVC dishes (104 $\mathrm{mm}$ diameter, $12 \mathrm{~cm}$ height). At the base of each dish there was a drainage outlet that was covered with strips of nylon mesh to allow excess water to be removed, while preventing the loss of sand. All samples were collected from the interspaces between shrubs in the revegetated area that was established in 1981 and transferred to the Water Balance Observation Site (about $1 \mathrm{~km}$ from the sampling site) at Shapotou Desert Research and Experiment Station, Chinese Academy of Sciences. Sampling was conducted in late February and late August, which was about 10 days before the experiments began in spring and autumn in 2013, respectively. Samples were gently processed and sprayed with distilled water to ensure that they were moist and that the sample structure remained intact. All samples were placed below the ground surface, with the top $2 \mathrm{~cm}$ left aboveground. Rain shelters were then placed at a height of $2 \mathrm{~m}$ above the samples. The soil surfaces surrounding the samples were paved with a straw curtain, which extended for $5 \mathrm{~m}$ beyond the shade of the shelters to prevent disturbance from sand particles outside the study area.

Both drought and sand burial stress treatments were conducted from 10 March (spring) and 1 September (autumn). A total of 108 samples were collected for each experiment 
in the two different seasons and were randomly divided into three water supply groups by applying distilled water in three artificial rainfall regimes at 8-day intervals in spring and autumn: 4 and $6 \mathrm{~mm}$ (average rainfall, control), 2 and $3 \mathrm{~mm}$ (double drought), and 1 and $1.5 \mathrm{~mm}$ (4-fold drought), respectively. To determine the effect of burial, six treatments were applied, with depths of 0 (control), 0.5, 1, 2, 4, and $10 \mathrm{~mm}$, equivalent to $0,4.25,8.5,17,34$, and $85 \mathrm{~mL}$ of dried sand, respectively. The sand was distributed gently and evenly over crusts that had been subjected to each of the water supply subgroups described above.

There were six replicates of the drought $\times$ sand burial treatment. The prescribed sand burial depths and quantities of water applied were selected based on actual sand burial depths and precipitation levels observed during the period of 19902010 (Li et al., 2012) in the study area. The duration of each experiment was 72 days.

\subsection{Measurements of the chlorophyll $a$ content, PSII photochemical efficiency, regeneration potential, and maximal shoot upgrowth}

On the day after each experiment was completed, the sand particles deposited over the moss were gently blown off, and the same weight of sand applied prior to the burial treatment was collected. The upper $26 \mathrm{~mm}$ (i.e., including the active moss rhizoids) and inner core $(5 \mathrm{~cm}$ diameter) were excavated from each original sample and placed into cylindrical plastic dishes ( $5 \mathrm{~cm}$ diameter, $28 \mathrm{~mm}$ height). Each dish had a drainage outlet at the bottom that was covered with a strip of nylon mesh to allow excess moisture to be removed. These smaller samples were more representative than the original samples because the edge effect of the PVC tube was removed.

The six small samples from each treatment were randomly divided into two subgroups: one for the determination of maximal shoot upgrowth, PSII photochemical efficiency, and regeneration potential and the other for the measurement of the chlorophyll $a$ content of $B$. argenteum. The methods used to measure the maximal shoot upgrowth, PSII photochemical efficiency, regeneration potential, and chlorophyll $a$ content of samples were adopted from Jia et al. (2012).

The maximal shoot elongation of B. argenteum was determined by the difference between the vertical distances from the upper edge of the PVC container to the uppermost part of the crust surface prior to sand burial and after removal from the sand at the end of the experiment using a Vernier caliper.

The samples were watered to saturation level and then cultured in a growth chamber (Thermoline Scientific Equipment Pty. Ltd, NSW, Australia). The photon flux density (PFD), air temperature (Ta), relative air humidity (RH), and $\mathrm{CO}_{2}$ concentration $(\mathrm{Ca})$ were set to $1000 \mathrm{mmol} \mathrm{m}^{-2} \mathrm{~s}^{-1}$, $25^{\circ} \mathrm{C}, 55 \%$, and $390 \mathrm{mmolm}^{-2} \mathrm{~s}^{-1}$, respectively, during the day (08:00-19:00 UTC+8), and $0 \mathrm{mmol} \mathrm{m}^{-2} \mathrm{~s}^{-1}, 15^{\circ} \mathrm{C}$, $65 \%$, and $400 \mathrm{mmol} \mathrm{m}^{-2} \mathrm{~s}^{-1}$, respectively, at night (19:00-
08:00 UTC +8 the next day). The position of each sample was randomly changed every day. After a 3-day preacclimation, the samples were wetted again to saturation level and PSII photochemical efficiency was measured $4 \mathrm{~h}$ later (when the maximum value occurred). The PSII photochemical efficiency $(\mathrm{Fv} / \mathrm{Fm})$ was determined by an analysis of the slow kinetics of chlorophyll fluorescence using a PAM-2000 fluorometer (Walz, Effeltrich, Germany). The device was adjusted to maintain a distance of $1.20 \mathrm{~cm}$ between the fiber optics exit plane and sample. Prior to measurement, the samples were dark adapted for $5 \mathrm{~min}$ and then supplemented with a sequence of weak irradiance and saturation pulses $\left(5000 \mathrm{mmol} \mathrm{m}^{-2} \mathrm{~s}^{-1}\right)$.

These samples were then hydrated, washed, and shaken to remove any sand attached. Then, $1 \mathrm{~mm}$ long portions of the upper shoots containing stem apices were isolated from each of the smaller samples and placed on native sand that had previously been sieved and dried as described by Stark et al. (2004). The same cylindrical plastic dishes as in the previous experiment were used, and the experimental conditions were also the same, except that moisture was supplied daily. The protonemal area was determined according to the protocols described by Barker et al. (2005) after a 58-day inoculation. The measurement of the regeneration potential of detached shoots was only conducted in the spring experiment, due to some of the autumn experiment samples being broken during transportation.

The chlorophyll $a$ content was determined on $\mathrm{a} \mathrm{mgg}^{-1}$ dry-weight basis by high-performance liquid chromatography (HPLC) using a method described by Gilmore and Yamamoto (1991). Briefly, $50 \mathrm{mg}$ of dry, soil-free shoots were collected from each sample, ground, extracted in $80 \%$ acetone, and then centrifuged at $10000 \mathrm{rpm}$ for $5 \mathrm{~min}$. After the removal of the supernatant, the remaining pellet was resuspended in $100 \%$ acetone and centrifuged again at $10000 \mathrm{rpm}$. The supernatants were then mixed and passed through a $20 \mathrm{~mm}$ filter prior to injection into a Spherisorb ODS 1 column (Alltech Associates Inc., Deerfield, IL, USA) at a flow rate of $1 \mathrm{~cm}$.

\subsection{Statistics}

Three-dimensional plots were produced using MatlabR2014a (The MathWorks Inc., Natick, MA, USA) to show the responses of chlorophyll $a$ content, PSII photochemical efficiency, regeneration potential, and shoot upgrowth of the biocrust moss $B$. argenteum to a combination of three levels of drought severity and six depths of sand burial.

A one-way analysis of variance (ANOVA) was used to test for any significant differences in the data using the SPSS 21 software (SPSS Inc., Chicago, USA). Differences between the individual parameters were evaluated by least significant difference (LSD) post hoc multiple comparisons at the $95 \%$ confidence level. 
Table 1. Changes in the percentage cover of Bryum argenteum Hedw. within a biocrust in response to sand burial depth in spring and autumn.

\begin{tabular}{lrr}
\hline Sand burial & \multicolumn{2}{c}{ Season } \\
\cline { 2 - 3 } depth $(\mathrm{mm})$ & \multicolumn{2}{c}{ Spring } \\
\hline 0 & $100.000 \pm 0.000 \mathrm{a}$ & $100.000 \pm 0.000 \mathrm{a}$ \\
0.5 & $53.556 \pm 0.882 \mathrm{c}$ & $67.960 \pm 0.923 \mathrm{~b}$ \\
1 & $22.667 \pm 2.915 \mathrm{e}$ & $28.433 \pm 0.308 \mathrm{~d}$ \\
2 & $0.000 \pm 0.000 \mathrm{~g}$ & $4.667 \pm 0.577 \mathrm{f}$ \\
4 & $0.000 \pm 0.000 \mathrm{~g}$ & $0.000 \pm 0.000 \mathrm{~g}$ \\
10 & $0.000 \pm 0.000 \mathrm{~g}$ & $0.000 \pm 0.000 \mathrm{~g}$ \\
\hline
\end{tabular}

Values are means $( \pm$ SE), different letters indicate significant differences between different sand burial depth treatments at the $p<0.05$ level as determined using an LSD post hoc test; $n=9$.

A detrended correspondence analysis (DCA) of the chlorophyll $a$ content, PSII photochemical efficiency, regeneration potential (protonemal area), and shoot upgrowth of $B$. argenteum was used to determine whether linear or unimodal ordination methods should be applied. We then performed a redundancy analysis (RDA) to determine the relationships between the parameters listed above and environmental parameters. A Monte Carlo permutation test $(n=$ 499) was used to determine the significance of all canonical axes. Both DCA and RDA were performed using Canoco for windows 5.0 (Ithaca, NY, USA).

\section{Results}

\subsection{Seasonal changes in $B$. argenteum cover and its response to sand burial depth}

Bryum argenteum can completely cover a soil surface, and there was no significant difference between spring and autumn cover when sand burial was absent (Table 1). Sand burial significantly reduced the $B$. argenteum cover in both seasons, with the amplitude decrease in autumn being significantly lower than that in spring (Table 1).

\subsection{Interactive effects of sand burial and drought on the chlorophyll $\boldsymbol{a}$ content of $\boldsymbol{B}$. argenteum}

The chlorophyll $a$ content of $B$. argenteum was generally lower in spring than under the same treatment in autumn, with the same trend found in the response to drought, sand burial, and their combination (Fig. 2). Drought uniformly imposed negative effects on the chlorophyll $a$ content, whereas burial by sand had a dual effect on the chlorophyll $a$ content (Fig. 2). The chlorophyll $a$ content increased in treatments when the burial depth was shallow $(<2 \mathrm{~mm})$ and decreased when the depth was larger (sand burial depth $\geq 2 \mathrm{~mm}$ ).

A significant interactive effect between drought and sand burial on the chlorophyll $a$ content of $B$. argenteum was found. Drought strengthened the positive effects of shallow burial and mediated the negative effects of deep burial with regard to chlorophyll $a$ retention (Fig. 2). In addition, sand burial weakened and even reversed the negative effects of drought on the retention of the chlorophyll $a$ content in B. argenteum (Fig. 2).

\subsection{Interactive effects of sand burial and drought on the PSII photochemical efficiency of $B$. argenteum}

The PSII photochemical efficiency of B. argenteum displayed the same trends as the chlorophyll $a$ content in response to drought, sand burial, and their combination, although it was generally lower in spring than under the same treatment in autumn (Fig. 3). Drought consistently exerted negative effects on the PSII photochemical efficiency, while burial by sand had a dual effect on the PSII photochemical efficiency (Fig. 3). The PSII photochemical efficiency increased in treatments where the burial depth was shallow $(<2 \mathrm{~mm})$ and decreased when the depth was larger (sand burial depth $\geq 2 \mathrm{~mm}$ )

A dramatic interactive effect between sand burial and drought on the PSII photochemical efficiency of $B$. argenteum was observed. Drought strengthened the positive effects of shallow burial and ameliorated the negative effects of deep burial with regard to PSII photochemical efficiency (Fig. 3). Sand burial diminished and even reversed the negative effects of drought on the retention of the PSII photochemical efficiency (Fig. 3).

\subsection{Interactive effects of sand burial and drought on the regeneration potential of detached shoots of B. argenteum}

Drought imposed negative effects on the regeneration potential of detached shoots of $B$. argenteum, while burial by sand had a dual effect on the regeneration potential (Fig. 4). The regeneration potential increased in treatments where the burial depth was shallow $(<2 \mathrm{~mm})$ and decreased when the depth was larger (sand burial depth $\geq 2 \mathrm{~mm}$ ).

There was a remarkable interactive effect of sand burial and drought on the regeneration potential of B. argenteum. Sand burial alleviated and even converted the negative effects of drought into positive effects with regard to the regeneration potential of detached shoots (Fig. 4). Drought enhanced the positive effects of shallow burial and eased the negative effects of deep burial on the regeneration potential of detached shoots (Fig. 4).

\subsection{Interactive effects of sand burial and drought on shoot elongation of $B$. argenteum}

Although $B$. argenteum shoots were generally less elongated in spring than under the same treatment in autumn, drought and sand burial had both negative and dual effects on shoot elongation, which was similar to the pattern observed for the 

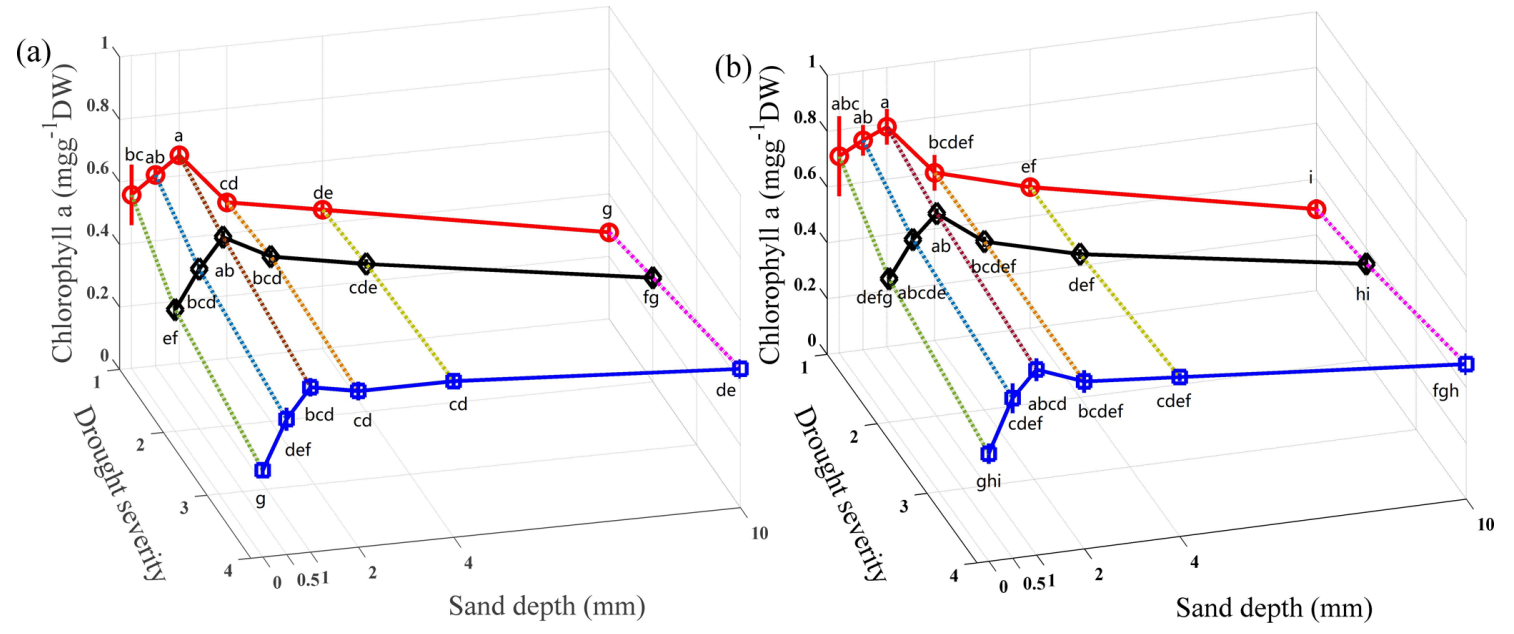

Figure 2. Changes in the chlorophyll a content of the biocrust moss Bryum argenteum Hedw. following exposure to natural precipitation (control; 1), half of the natural precipitation amount (2), and one-fourth of the natural precipitation amount (4), combined with 0 (control), $0.5,1,2,4$, and $10 \mathrm{~mm}$ depth of sand burial in spring (a) and autumn (b). Symbols represent means \pm SE. Different letters indicate significant differences between different drought severities and sand burial depth treatments at the $p<0.05$ level, as determined using an LSD post hoc test.
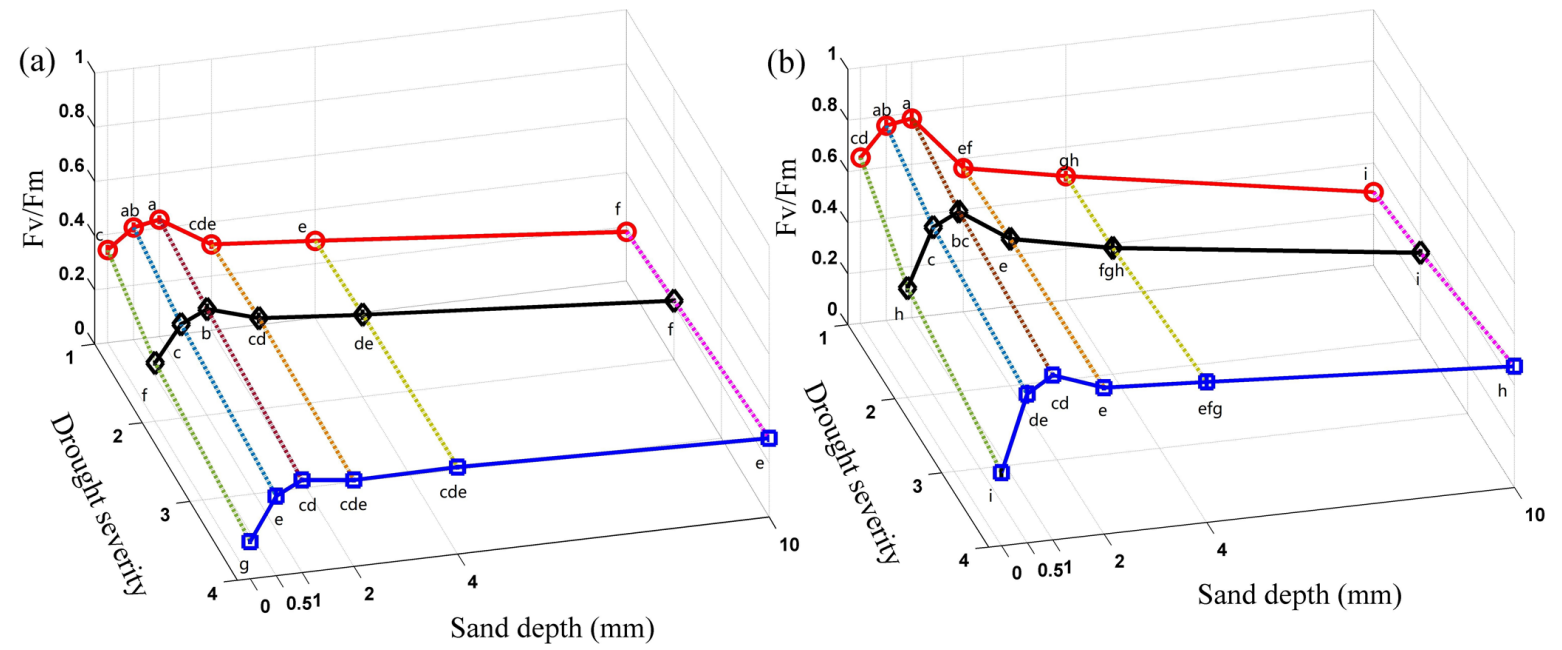

Figure 3. Changes in the PSII photochemical efficiency (Fv/Fm) of the biocrust moss Bryum argenteum Hedw. following exposure to natural precipitation (control; 1), half of the natural precipitation amount (2), and one-fourth of the natural precipitation amount (4), combined with 0 (control), 0.5, 1, 2, 4, and $10 \mathrm{~mm}$ depth of sand burial in spring (a) and autumn (b). Symbols represent means \pm SE. Different letters indicate significant differences between different drought severities and sand burial depth treatments at the $p<0.05$ level as determined using an LSD post hoc test.

other three parameters described above (Fig. 5). Conversely, drought reduced the positive effects of shallow burial and exacerbated the negative effects of deep burial on shoot upgrowth. In addition, sand burial aggravated the negative effects of drought on shoot elongation (Fig. 5).

\subsection{Redundancy analysis of the combined effects of sand burial and drought on the chlorophyll $a$ content, PSII photochemical efficiency, regeneration potential, and shoot upgrowth of B. argenteum}

The RDA analysis results showed that drought was a more important stressor influencing shoot elongation than sand burial (Fig. 6), while sand burial played a more important role in the retention of the chlorophyll $a$ content, PSII photo- 


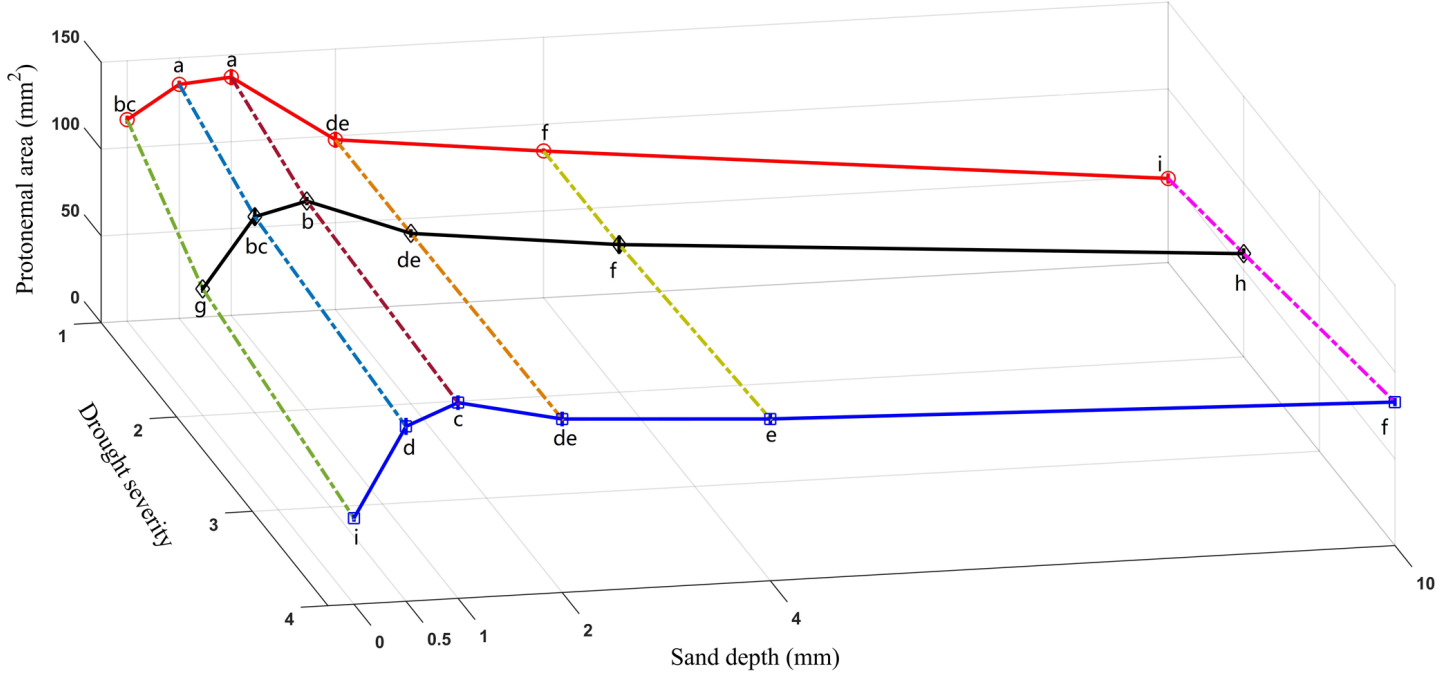

Figure 4. Changes in the protonemal area of detached shoots of the biocrust moss Bryum argenteum Hedw. following exposure to natural precipitation (control; 1), half of the natural precipitation amount (2), and one-fourth of the natural precipitation amount (4), combined with 0 (control), $0.5,1,2,4$, and $10 \mathrm{~mm}$ depth of sand burial in spring. Symbols represent means \pm SE. Different letters indicate significant differences between different drought severities and sand burial depth treatments at the $p<0.05$ level as determined using an LSD post hoc test.

(a)

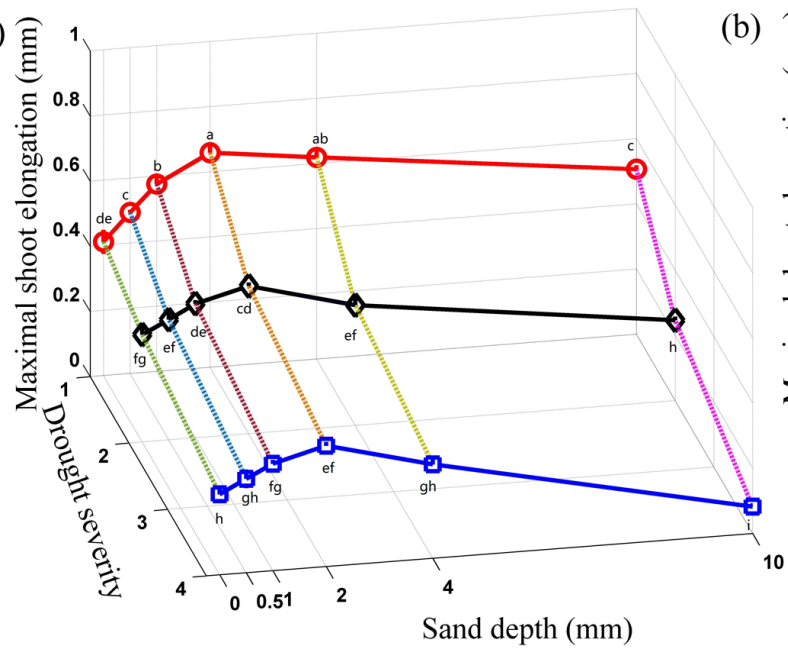

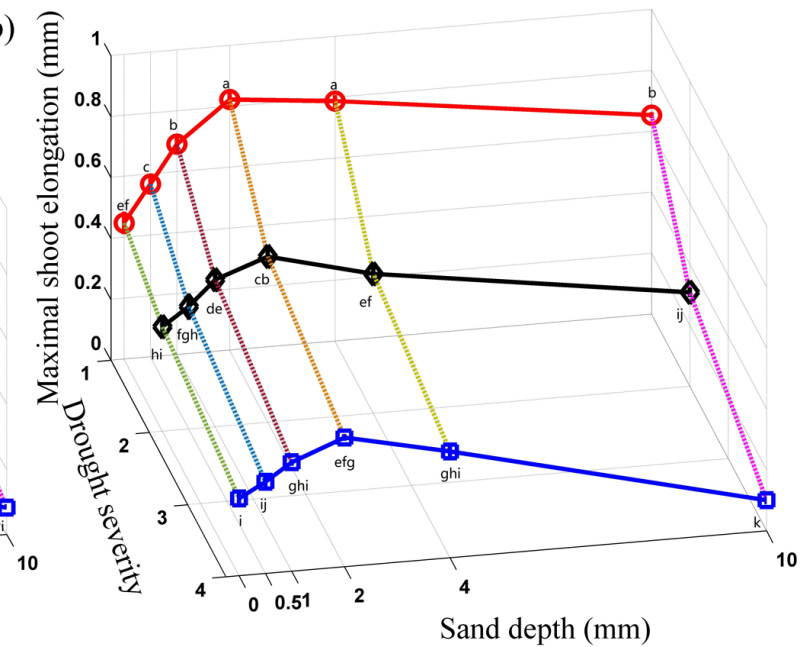

Figure 5. Changes in the maximal shoot elongation of the biocrust moss Bryum argenteum Hedw. following exposure to natural precipitation (control; 1), half of the natural precipitation amount (2), and one-fourth of the natural precipitation amount (4), combined with 0 (control), $0.5,1,2,4$, and $10 \mathrm{~mm}$ depth of sand burial in spring (a) and autumn (b). Symbols represent means \pm SE. Different letters indicate significant differences between different drought severities and sand burial depth treatments at the $p<0.05$ level as determined using an LSD post hoc test.

chemical efficiency, and regeneration potential than drought. Specifically, sand burial was positively correlated with the chlorophyll $a$ content, PSII photochemical efficiency, regeneration potential, and shoot elongation when the burial depth was shallow ( $<2 \mathrm{~mm}$; Fig. 6a), while it was negatively correlated with the four variables when the depth was larger (sand burial depth $\geq 2 \mathrm{~mm}$; Fig. $6 \mathrm{~b}$ ). In addition, drought was negatively correlated with the four variables when the burial depth was shallow ( $<2 \mathrm{~mm}$; Fig. 6a) but positively correlated with all variables, except for shoot elongation, when the depth was larger (sand burial depth $\geq 2 \mathrm{~mm}$; Fig. $6 \mathrm{~b}$ ).

The four parameters investigated in this study were more readily affected by sand burial and drought in autumn than in spring when the burial depth was shallow ( $<2 \mathrm{~mm}$; Fig. 6a). Under deep-sand burial (sand burial depth $\geq 2 \mathrm{~mm}$; Fig. 6b), the chlorophyll $a$ content, PSII photochemical efficiency, and regeneration potential were more sensitive to sand burial in autumn than in spring, but shoot elongation was more sus- 
ceptible to sand burial in spring than autumn. In contrast, the chlorophyll $a$ content, PSII photochemical efficiency, and regeneration potential were more sensitive to drought in spring than in autumn, while shoot elongation was more susceptible to sand burial in autumn than spring under the deep-sand burial treatments (sand burial depth $\geq 2 \mathrm{~mm}$; Fig. $6 \mathrm{~b}$ ).

\section{Discussion}

A desert is a multi-stressed environment, generally characterized by a series of stressors (Xie et al., 2007; Powell et al., 2015). The biocrust moss, Bryum argenteum Hedw. generally acts as a pioneer, and even dominant species, inhabiting many desert ecosystems due to its high resistance and versatile adaptation strategies to stressors (Li et al., 2014; Weber et al., 2016). There is growing evidence that biocrust organisms, including mosses, are extremely vulnerable to stressors originating mostly from climate change and disturbances (Reed et al., 2012; Weber et al., 2016). Ferrenberg et al. (2015) found that climate change and physical disturbances may cause similar community shifts within biocrusts. In arid sandy desert ecosystems, drought and sand burial are the two prevailing stressors and are induced separately by climate change and disturbance. They act as filters, eliminating the sensitive species by determining the physiology, growth, and survival of biocrust mosses (Martínez and Maun, 1999; Barker et al., 2005; Jia et al., 2008). In this study, we found that drought and sand burial exerted different, but dual effects on the physiology and growth of B. argenteum. More interestingly, both antagonistic and additive effects of drought and sand burial on B. argenteum were observed (Fig. 6), which explained the survival and distribution of B. argenteum in an arid sandy desert where the two stressors can occur simultaneously.

\subsection{Mutually antagonistic effects between drought and sand burial enable $B$. argenteum to survive the co-occurrence of the two stressors in an arid sandy desert}

As hypothesized, it was found that a combination of sand burial and drought did not always exacerbate the individual negative effects of each stressor on $B$. argenteum. Drought significantly ameliorated the negative effects of deep-sand burial on PSII photochemical efficiency (Fig. 2), the retention of chlorophyll $a$ content (Fig. 3), and regeneration potential (Fig. 4) of B. argenteum. Sand burial diminished and even reversed the negative effects of drought on the maintenance of the chlorophyll $a$ content (Fig. 2), PSII photochemical efficiency (Fig. 3), and regeneration potential (Fig. 4) of $B$. argenteum. These mutually antagonistic effects on the physiological vigor of the biocrust moss provided an opportunity for it to overcome the two co-occurring stressors, and this may be an important reason why it usually acts as the pioneer moss species by colonizing and even flourishing on the ground surface throughout China's sandy deserts.

The antagonistic effects of these two stressors are shortterm physiological indicators, implying that $B$. argenteum has a strong potential to photosynthesize or regenerate after their removal. It is difficult for the moss to maintain this potential for a long time due to the increased use or exhaustion of its stored carbohydrate reserves when buried (Maun, 1998; Kent et al., 2005). Therefore, other long-term parameters (e.g., growth rate) also need to be considered.

\subsection{Additive negative effects between drought and sand burial limit the distribution of $B$. argenteum in an arid sandy desert}

In contrast to our expectations, the mutually antagonistic effects of drought and sand burial did not impact on long-term shoot upgrowth, even though sand burial (depth $\leq 4 \mathrm{~mm}$ ) stimulated shoot elongation (Fig. 5). This additive negative effect inflicted by the combination of drought and sand burial on shoot upgrowth suggested a trade-off between growth and stress tolerance (Steinberg, 2012). In general, there is a tradeoff between growth and physiological vigor, including regeneration potential, when the moss is exposed to stress. This is in accordance with the theory that adaptation to stress carries a cost, and spending resources on defense or resistance leads to a weakened performance in conditions where these traits are not needed (Bijlsma and Loeschcke, 2005). Collectively, the preservation of physiological activity (photosynthetic pigment, PSII photochemical efficiency) and the propagation of fecundity (protonemal area), afforded by the mutually antagonistic effects, at the cost of shoot elongation caused by the negative additive effect, under long-term, deep-sand burial, will result in the failure of B. argenteum shoots to protrude above the sand surface. This could even lead to death, ultimately causing the moss to vanish from the ecosystem. This can explain the absence of B. argenteum in areas suffering from long-term, deep-sand burial stress, such as flowing sand or seriously degraded landscapes. It also explains why $B$. argenteum can colonize soil surfaces only after the burial depth decreases to a shallow level, through ecological construction and restoration measures throughout the arid sandy areas of northern China.

Based on a conceptual model, Bowker et al. (2006) and Li et al. (2010) both proved that biocrust moss is restricted to a specific topography, where it is less stressed by the microclimate and disturbances than at other positions on microspatial scales. However, the distribution of B. argenteum is apparently vaster and more continuous than that indicated by Bowker et al. (2006) and Li et al. (2010) in arid sandy areas, where sand burial is pervasive and occurs regularly. Thus, it is suggested that the interaction between physical environmental stressors from resource limitation, climate, and physical disturbances can be used to facilitate an extension of the ecological niche of desert moss (Callaway, 1995). Therefore, 
(a)

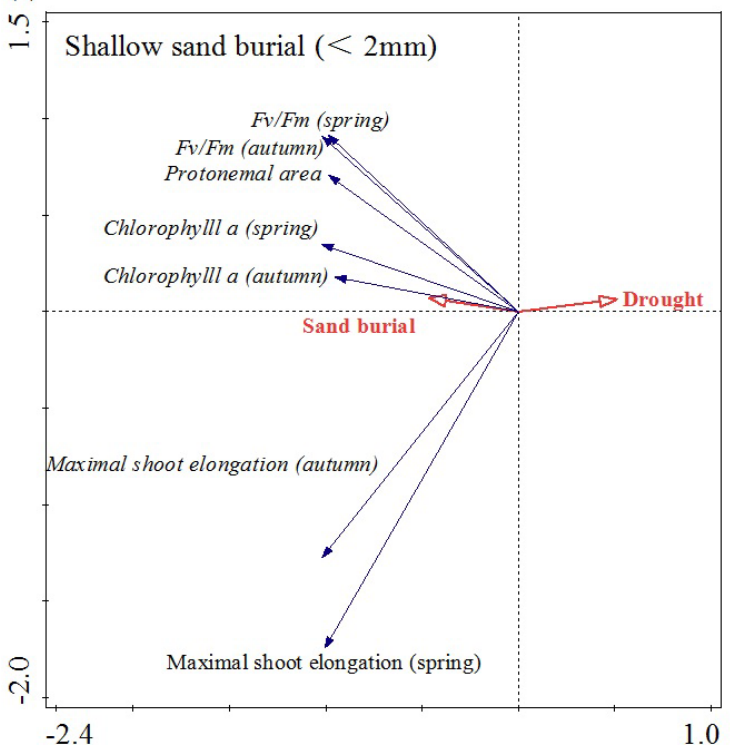

(b)

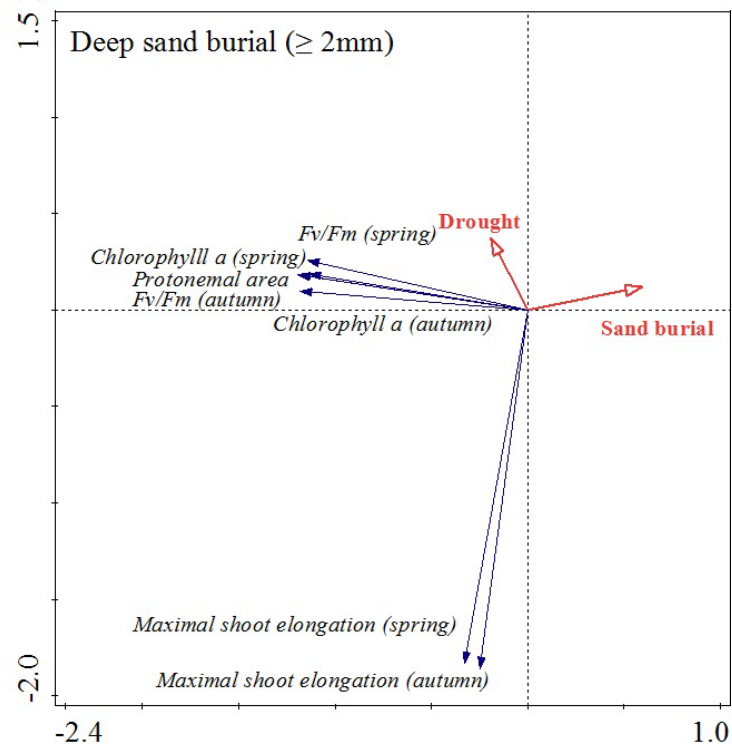

Figure 6. RDA diagram of the effect of drought, sand burial, and their combination on the chlorophyll $a$ content, PSII photochemical efficiency $(\mathrm{Fv} / \mathrm{Fm})$, regeneration potential (protonemal area), and shoot upgrowth (maximal shoot elongation) of biocrust moss Bryum argenteum Hedw. Under a shallow sand burial treatment, the eigenvalues were 0.8134 and 0.0152 for the first and second axes, respectively, and the correlation coefficients were 0.9527 and 0.4876 , respectively. In terms of deep-sand burial, the eigenvalues of the first and second axes were 0.6068 and 0.2379 , respectively, and the correlation coefficients were 0.9362 and 0.9126 , respectively. The Monte Carlo permutation test indicates that all variables were significantly correlated with the environmental factors $(p<0.05)$.

corresponding models should take into account the interactions between climate change and physical disturbances, and from an evolutionary perspective the environmental pressure and biological response should be considered integratively. This study also found that biocrust moss could be harmed by climate change, with conditions such as drought predicted to be more frequent and extensive in the future. This damage may be alleviated by other environmental factors or disturbances, such as sand burial, although this needs to be verified further.

\subsection{Possible mechanisms underlying the combined effects of drought and sand burial on $B$. argenteum and its significance in ecological construction}

It is not fully understood why drought and sand burial exert antagonistic effects on the physiological activity and asexual propagating fecundity of B. argenteum. It is possible that the antagonistic effect may originate from the water-conserving effect of sand burial, which could mitigate the negative effect of drought to some extent (Meng et al., 2011). For over 300 years in China, sand burial has been widely used by farmers as a useful moisture-conserving measure to cultivate crops, in a practice referred to as Shatian or sandy field ( $\mathrm{Li}$ et al., 2000). However, this beneficial effect has rarely been reported for biocrust mosses. In addition, sand burial can also provide a protective shell for $B$. argenteum, mitigating the damage from other stresses, such as wind (Liu et al., 2013).
On the other hand, drought favors $B$. argenteum under sand burial by lowering the risk of carbon starvation induced by the reduction in photosynthetic area and the relatively trivial rainfall (causing the partial hydration of moss). This favorable effect of drought has also been reported for bundled filamentous cyanobacteria following sand burial (Williams, 2011; Rao et al., 2012) or thermal stress (Lan et al., 2014) and for mosses exposed to heat shock (Xu et al., 2009), ultraviolet-B (Turnbull et al., 2009), and fungal attack (Weber et al., 2016). Drought would increase the removal rate of sand by enabling the dry sand to be more easily blown by wind, resulting in a harmful deep burial becoming a beneficial shallow burial. Therefore, drought is also considered to have a dual effect, especially when deep-sand burial occurs.

The ability to achieve a higher rate of shoot elongation gives $B$. argenteum an important advantage over other moss species, enabling it to rapidly recover from sand burial (Jia et al., 2008). However, shoot elongation in B. argenteum is severely inhibited by drought, due to the lower amounts (Jia et al., 2008) and/or shorter durations (Kidron et al., 2010) of moisture availability. This could be interpreted as a reduction in the accumulation of carbohydrate gained by photosynthesis or as carbon starvation (Barker et al., 2005) caused by drought. Sand burial not only directly reduces the photosynthetic area of mosses but also causes a deterioration in the environmental conditions required for photosynthesis (e.g., the reduction in photosynthetically active radiation and 
blocking gas exchange). Furthermore, the sand deposited on $B$. argenteum would intercept water from precipitation, decreasing the quantity of rainfall available to the moss, with the trivial amount of precipitation received already identified as being detrimental (inducing carbon starvation) to biocrust moss (Alpert and Oechel, 1985; Belnap et al., 2004). This would consolidate the negative effect of drought on the shoot elongation of $B$. argenteum.

In recent years, the rapid artificial cultivation of biocrust has provided a novel alternative to traditional biological methods for controlling erosion (Bu et al., 2014; Doherty et al., 2015; Antoninka et al., 2016). At the same time, biocrust moss is considered to be a potentially promising biological material that could be inoculated to accelerate the process of sand fixation and the recovery of degraded soil (Antoninka et al., 2016). However, this technique is still limited to laboratory trials, with no successful large-scale application in the field reported. One key reason for this is that the moss typically occupies the late successional stage among biocrusts and its environmental requirements are high. The moss cultured in the laboratory under favorable conditions cannot withstand the unfavorable stress from drought, high temperature, and UV-B exposure. However, the results of this study indicate that moderate sand burial may have the potential to alleviate these stresses and increase the survival ratio of artificially cultured biocrust moss in the restoration of arid sandy deserts or degraded ecosystems. The use of such a technique is also in agreement with Maestre et al. (2006), who found that the inoculation of biocrusts in the form of slurry combined with the addition of composted sewage sludge, which has a similar effect to that of burial, encouraged the recovery of biocrust in degraded soils from semiarid Mediterranean areas.

\subsection{Seasonal effects on the combined effects of drought and sand burial on $B$. argenteum}

Coverage (Table 1), physiological vigor (Figs. 2 and 3), and growth rate (Fig. 4) of B. argenteum and the response to drought and sand burial varied with season (Fig. 5). In our study area, the physiological activity of $B$. argenteum reached its lowest level after a long-term cold and drought stress in winter (Li et al., 2012). Conserving its activity in the continuous drought of spring enabled the moss to be ready to obtain more carbon through photosynthesis in the relatively favorable conditions in summer (higher precipitation). In autumn, when the physiological activity of $B$. argenteum was highest, it is essential to gain height through shoot upgrowth to cope with the following sand burial in winter. This successful seasonal adaptation strategy of $B$. argenteum to the co-occurring stressors of drought and sand burial was supported by our results. Therefore, the seasonal distribution of precipitation and sand burial in our study area was important because it enabled $B$. argenteum to be the pioneer species in our study area, and this mechanism may be valid in areas suffering from the co-occurring stressors of drought and sand burial in sandy deserts elsewhere in China and worldwide.

Data availability. No data sets were used in this article.

Competing interests. The authors declare that they have no conflict of interest.

Special issue statement. This article is part of the special issue " $\mathrm{Bi}$ ological soil crusts and their role in biogeochemical processes and cycling". It is a result of the BIOCRUST3 conference, Moab, USA, 26 to 30 September 2016.

Acknowledgements. We gratefully acknowledge Bettina Weber, David Elliott, and one anonymous reviewer for their invaluable comments on the manuscript. This research was partially supported by the Natural Science Foundation of China (grant nos. 41371099, 41671210, 41621001) and CAS "Light of West China” Program.

Edited by: Bettina Weber

Reviewed by: David Elliott and one anonymous referee

\section{References}

Alpert, P. and Oechel, W. C.: Carbon balance limits microdistribution in the desiccation-tolerant plant, Grimmia laevigata, Ecology, 66, 660-669, 1985.

Antoninka, A., Bowker, M. A., Reed, S. C., and Doherty, K.: Production of greenhouse-grown biocrust mosses and associated cyanobacteria to rehabilitate dryland soil function, Restor. Ecol., 24, 324-35, 2016.

Barker, D. H., Stark, L. R., Zimpfer, J. F., Mcletchie, N. D., and Smith, S. D.: Evidence of drought-induced stress on biotic crust moss in the Mojave Desert, Plant Cell Environ., 28, 939-947, 2005.

Belnap, J. and Gillette, D. A.: Vulnerability of desert biological soil crusts to wind erosion: the influences of crust development, soil texture, and disturbance. J. Arid Environ., 39, 133-142, 1998.

Belnap, J., Phillips, S. L., and Miller, M. E.: Response of desert biological soil crusts to alterations in precipitation frequency, Oecologia, 141, 306-316, https://doi.org/10.1007/s00442-0031438-6, 2004.

Bijlsma, R. and Loeschcke, V.: Environmental stress, adaptation and evolution: an overview, J. Evol. Biol., 18, 744-749, 2005.

Bowker, M. A., Belnap, J., Davidson, D. W., and Goldstein, H.: Correlates of biological soil crust abundance across a continuum of spatial scales: support for a hierarchical conceptual model, J. Appl. Ecol., 43, 152-163, 2006.

$\mathrm{Bu}$, C., Wu, S., Yang, Y., and Zheng, M.: Identification of factors influencing the restoration of cyanobacteriadominated biological soil crusts, PLoS ONE, 9, e90049, https://doi.org/10.1371/journal.pone.0090049, 2014. 
Callaway, R. M.: Positive interactions among plants, Bot. Rev., 61, 306-349, 1995.

Doherty, K. D., Antoninka, A. J., Bowker, M. A., Ayuso, S. V., and Johnson, N. C.: A novel approach to cultivate biocrusts for restoration and experimentation, Ecol. Restor., 33, 13-16, 2015.

Ferrenberg, S., Reed, S. C., and Belnap, J.: Climate change and physical disturbance cause similar community shifts in biological soil crusts, P. Natl. Acad. Sci. USA, 112, 12116-12121, 2015.

Garcia-Pichel, F. and Belnap, J.: Microenvironments and microscale productivity of cyanobacterial desert crusts, J. Phycol., 32, 774782, 1996

Gilmore, A. M. and Yamamoto, H. Y.: Resolution of lutein and zeaxanthin using a non-endcapped, lightly carbon-loaded C18 high-performance liquid chromatographic column, J. Chromatogr., 35, 137-145, 1991.

Huxman, T. E., Snyder, K. A., Tissue, D. Leffler, A. J., Ogle, K., Pockman, W. T., Sandquist, D. R., Potts, D. L., and Schwinning, S.: Precipitation pulses and carbon fluxes in semiarid and arid ecosystems, Oecologia, 141, 254-268, https://doi.org/10.1007/s00442-004-1682-4, 2004.

IPCC: Climate Change: the Physical Science Basis: Working Group I Contribution to the Fourth Assessment Report of the Intergovernmental Panel on Climate Change, Cambridge University Press, Cambridge, 2007.

Jia, R. L., Li, X. R., Liu, L. C., Gao, Y. H., and Li, X. J.: Responses of biological soil crusts to sand burial in a revegetated area of the Tengger Desert, Northern China, Soil Biol. Biochem., 40, $2827-$ 2834, 2008.

Jia, R. L., Li, X. R., Liu, L. C., Gao, Y. H., and Li, X. T.: Differential wind tolerance of soil crust mosses explains their microdistribution in nature, Soil Biol. Biochem., 45, 31-39, 2012.

Kent, M., Owen, N. W., and Dale, M. P.: Photosynthetic responses of plant communities to sand burial on the machair dune systems of the Outer Hebrides, Scotland, Ann. Bot.-London, 95, 869-877, https://doi.org/10.1093/aob/mci093, 2005.

Kidron, G. J., Vonshak, A., and Abeliovich, A.: Microbiotic crusts as biomarkers for surface stability and wetness duration in the Negev Desert, Earth Surf. Proc. Land., 34, 1594-1604, 2010.

Lan, S. B., Wu, L., Zhang, D. L., and Hu, C. X.: Desiccation provides photosynthetic protection for crust cyanobacteria $\mathrm{Mi}$ crocoleus vaginatus from high temperature, Physiol. Plantarum, 152, 345-354, https://doi.org/10.1111/ppl.12176, 2014.

Li, J. H., Li, X. R., and Zhang, P.: Micro-morphology, ultrastructure and chemical composition changes of Bryum argenteum from a desert biological soil crust following one-year desiccation, Bryologist, 117, 232-240, 2014.

Li, X. R.: Eco-Hydrology of Biological Soil Crusts in Desert Region of China, Higher Education Press, Beijing, 2012.

Li, X. Y., Gong, J. D., Gao, Q. Z., and Wei, X. H.: Rainfall interception loss by pebble mulch in the semiarid region of China, $\mathrm{J}$. Hydrol., 228, 165-173, 2000.

Li, X. R., Xiao, H. L., Zhang, J. G., and Wang, X. P.: Longterm ecosystem effects of sand-binding vegetation in the Tengger Desert, northern China, Restor. Ecol., 12, 376-390, 2004.

Li, X. R., He, M. Z., Zerbe, S., Li, X. J., and Liu, L. C.: Microgeomorphology determines community structure of biological soil crusts at small scales, Earth Surf. Proc. Land., 35, 932-940, 2010.
Li, X. R., Zhang, P., Su, Y. G., and Jia, R. L.: Carbon fixation by biological soil crusts following revegetation of sand dunes in arid desert regions of China: A four-year field study, Catena, 97, 119$26,2012$.

Liu, B., Liu, Z. M., Lü, X. T., Maestre, F. T., and Wang, L. X.: Sand burial compensates for the negative effects of erosion on the dune-building shrub Artemisia wudanica, Plant Soil, 374, 263273, https://doi.org/10.1007/s11104-013-1866-y, 2013.

Maestre, F. T., Martín, N., Díez, B., López-Poma, R., Santos, F., Luque, I., and Cortina, J.: Watering, fertilization, and slurry inoculation promote recovery of biological crust function in degraded soils, Microb. Ecol., 52, 365-377, 2006.

Martínez, M. L. and Maun, M. A.: Responses of dune mosses to experimental burial by sand under natural and greenhouse conditions, Plant Ecol., 145, 209-219, 1999.

Maun, M. A.: Adaptations of plants to burial in coastal sand dune systems, Can. J. Bot., 7, 713-738, 1998.

Maun, M. A.: Burial of plants as a selective force in sand dunes, in: Coastal Dunes: Ecology and Management, edited by: Martinez, M. L., Psuty, N. P., Springer-Verlag Berlin Heidelberg, 119-136, 2008.

Meng, J., Bu, C. F., Zhang, X. C., and Sun, Z. F.: Effects of biological crust removal and sand mulching on soil evaporation in a sandy soil, Bull. Soil Water Conserv., 31, 58-62, 2011 (in Chinese with English abstract).

Powell, J. T., Chatziefthimiou, A. D., Banack, S. A., Cox, P. A., and Metcalf, J. S.: Desert crust microorganisms, their environment, and human health, J. Arid Environ., 112, 127-133, 2015.

Rao, B. Q., Liu, Y. D., Lan, S. B., Wu, P. P., Wang, W. B., and Li, D. H.: Effects of sand burial stress on the early developments of cyanobacterial crusts in the field, Eur. J. Soil Biol., 48, 48-55, 2012.

Reed, S. C., Coe, K. K., Sparks, J. P., Housman, D. C., Zelikova, T. J., and Belnap, J.: Changes to dryland rainfall result in rapid moss mortality and altered soil fertility, Nat. Clim. Change, 2, 752-755, 2012.

Smith, M. D.: An ecological perspective on extreme climatic events: a synthetic definition and framework to guide future research, J. Ecol., 99, 656-663, 2011.

Stark, L. R., Nichols, L., McLetchie, D. N., Smith, S. D., and Zundel, C.: Age and sex specific rates of leaf regeneration in the Mojave Desert moss Syntrichia caninervis, Am. J. Bot., 91, 19, 2004

Steinberg, C. E. W.: Stress Ecology: Environmental Stress as Ecological Driving Force and Key Player in Evolution, https://doi.org/10.1007/978-94-007-2072-5, Springer, Berlin, 2012.

Turnbull, J. D., Leslie, S. J., and Robinson, S. A.: Desiccation protects Antarctic mosses from ultraviolet-B induced DNA damage, Funct. Plant Biol., 36, 214-221, 2009.

Weber, B., Büdel, B., and Belnap, J. (Eds.): Biological soil crusts: an organizing principle in drylands, in: Ecological Studies, vol. 226, https://doi.org/10.1007/978-3-319-30214-0, Springer, Springer International Publishing, Switzerland, 2016.

Whitford, W. G.: Ecology of Desert Systems, Academic Press, London, 2002.

Williams, W. J. and Eldridge, D. J.: Deposition of sand over a cyanobacterial soil crust increases nitrogen bioavailability in a semi-arid woodland, Appl. Soil Ecol., 49, 26-31, 2011. 
Xie, Z., Liu, Y., Hu, C., Chen, L., and Li, D.: Relationships between the biomass of algal crusts in fields and their compressive strength, Soil Biol. Biochem., 39, 567-572, 2007.

Xu, S. J., Liu, C. J., Jiang, P. A., Cai, W. M., and Wang, Y.: The effects of drying following heat shock exposure of the desert moss Syntrichia caninervis, Sci. Total Environ., 407, 2411-2419, 2009.
Zhang, Y. M., Wu, N., Zhang, B. C., and Zhang, J.: Species composition, distribution patterns and ecological functions of biological soil crusts in the Gurbantunggut Desert, J. Arid Land, 2, 180189, 2010. 